\title{
An Architecture for the Seamless Integration of UAS Remote Sensing Missions
}

\author{
E. Pastor, C. Barrado, P. Royo, J. Lopez, E. Santamaria*and X. Prats ${ }^{\dagger}$ \\ Technical University of Catalonia, Barcelona, Spain
}

\begin{abstract}
Unmanned Aerial Systems (UAS) are slowing becoming efficient platforms that can be applied in scientific/commercial remote sensing applications. UAS may offer interesting benefits in terms of cost, flexibility, endurance, etc. On the other side, the complexity of developing a full UAS-system is currently limiting its practical application. Currently, only large organizations like NASA or NOAA have enough budget and infrastructure to develop such applications.

Nowadays, UAS technology offers feasible technical solutions for airframes, flight control, communications, and a wide set of multi-spectrum sensors. However, the generalized development of remote sensing applications are still limited by the absence of systems that support the development of the actual UAS sensing mission.

This paper introduces a flexible and reusable hardware/software architecture designed to facilitate the development of UAS-based remote sensing applications. This flexibility is organized into an user-parameterizable UAS Service Abstraction Layer (USAL). The USAL defines a collection of standard services are their interrelations as a basic starting point for further development by users. Functionalities like enhanced flight-plans, a mission control engine, data storage, communications management, etc. are offered. Additional services can be included according to requirements but all existing services and inter-service communication infrastructure can be exploited and tailored to specific needs. The overall USAL architecture is demonstrated by means of a wild land fire remote sensing application currently being developed to support fire fighters in the Mediterranean area.
\end{abstract}

\section{Nomenclature}

UAS Unmanned Aircraft System

$U A V \quad$ Unmanned Aerial Vehicle

USAL UAS Service Abstraction Layer

FPM Flight Plan Manager

$R N A V \quad$ Area Navigation

$X M L \quad$ Extensible Markup Language

$S O A \quad$ Service Oriented Architecture

$L A N \quad$ Local Area Network

$V A S \quad$ Virtual Autopilot Service

GIS Geographical Information System

\footnotetext{
*Computer Architecture Dept., Avda. del Canal Olímpic 15, 08860 Castelldefels, Spain.

${ }^{\dagger}$ Castelldefels School of Technology, Avda. del Canal Olímpic 15, 08860 Castelldefels, Spain.
} 


\section{Introduction}

Current Unmanned Aerial System (UAS) technology offers feasible technical solutions for airframes, flight control, communications, and base stations. In addition, the evolution of technology is miniaturizing most sensors used in airborne applications. Hence, sensors like weather radars, SAR, multi spectrum linescan devices, etc. in addition to visual and thermal cameras are being used as payload on board UAS. As a result UAS are slowly becoming efficient platforms that can be applied in scientific/commercial remote sensing applications. UAS may offer interesting benefits in terms of cost, flexibility, endurance, etc. Even remote sensing in dangerous situations due to extreme climatic conditions (wind, cold, heat) are now seen as possible because the human factor on board the airborne platform is no longer present. On the other side, the complexity of developing a full UAS-system tailored for remote sensing is currently limiting its practical application. Currently, only large organizations like NASA ${ }^{1}$ or $^{2,3}$ NOAA have enough budget and infrastructure to develop such applications, and eventually may lease flight time to other organizations to conduct their experiments.

Even though the rapid evolution of UAS technology on airframes, autopilots, communications and payload (see these components in Figure 1), the generalized development of remote sensing applications is still limited by the absence of systems that support the development of the actual UAS sensing mission. Remote sensing engineers face the development of specific systems to control their desired flight-profile, sensor activation/configuration along the flight, data storage and eventually its transmission to the ground control. All this elements may delay and increase the risk and cost of the project. If realistic remote sensing applications should be developed, additional support to effective system support must be created to offer flexible and adaptable platforms for any application that is susceptible to use them.

This paper introduces a flexible and reusable hardware/software architecture designed to facilitate the development of UAS-based remote sensing applications. Over a set of embedded microprocessors (both in the UAS and the ground control station) we build a distributed embedded system connected by a local area network. Applications are developed following a service/subscription based software architecture. Each computation module may support multiple applications. Each application could create and subscribe to available services. Services could be discovered and consumed in a dynamic way like web services in the Internet domain. Applications could interchange information transparently from network topology, application implementation and actual data payload.

This flexibility is organized into an user-parameterizable UAS Service Abstraction Layer (USAL). The USAL defines a collection of standard services are their interrelations as a basic starting point for further development by users. Functionalities like enhanced flight-plans, a mission control engine, data storage, communications management, etc. are offered. Additional services can be included according to requirements but all existing services and inter-service communication infrastructure can be exploited and tailored to specific needs. This approach reduces development times and risks, but at the same time gives the user higher levels of flexibility and permits the development of more ambitious applications.

As application scenario, our objective is to develop support for general remote sensing applications, but in particular we are interested in a UAS system devoted to the detection, control and analysis of wild land forest fires in the Mediterranean area. Real time information obtained by this aerial platform together with its analysis could be shared with fire brigades on the ground will allow fire chiefs take the strategic decisions to optimize actions against the fire, thus reducing damage to the environment, costs, and improving the security of the overall operations.

This paper is organized as follows. SectionII motivates the selection of UAS as a new family of remote sensing platforms. Section III generally describes the USAL architecture and the underlying service oriented technologies that will be applied to UAS avionics. Section IV details the most relevant services that are included in the USAL to facilitate the development of UAS applications. Section V details the UAS-based wildland fire monitoring application and in particular the so called hot spot mission, detailing to some extent those services and data flows involved. Finally, Section VII concludes the paper and outlines future research and development directions.

\section{Motivation}

Remote sensing makes it possible to collect data on dangerous or inaccessible areas and to replace costly data collection on the ground. Also, aerial remote sensing ensures surveillance tasks without disturbing the 


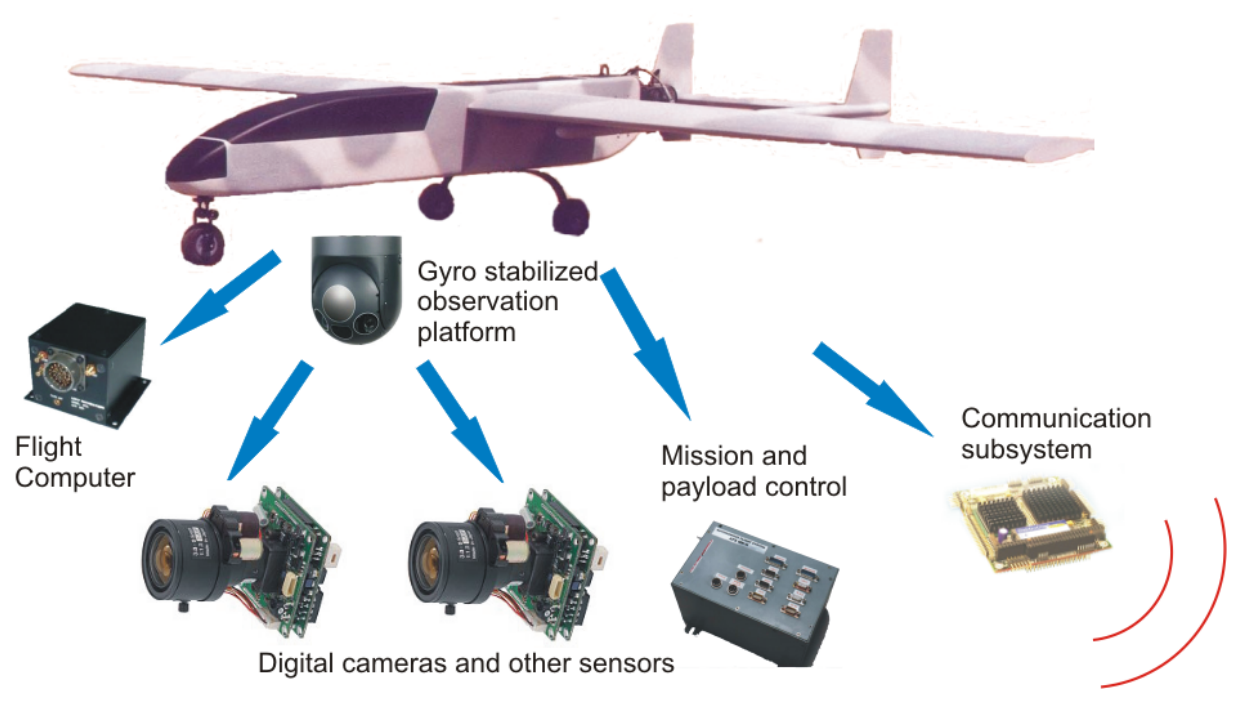

Figure 1. Schematic view of a UAS sensor platform

area conditions. Civil applications of remote sensing include monitoring forests, study the effects of climate change, natural resource management, agricultural fields usage and conservation, and national security on border areas.

The remote sensing discipline relates with many scientific and technological research fields. But the development of aircraft technology first, ${ }^{4}$ and of artificial satellites latter, ${ }^{5}$ allowed remote sensing to progress to a global scale. Nowadays, the UAS new flight technology is emerging as a new tool for remote sensing. Many types of UAS exist today; however the class of tactical/mini UAS is emerging as the valid new option for remote sensing. This UAS "explosion follows the same evolution of aircrafts and satellites. In the same way that aircraft and satellite were initially developed for military purposes during the Cold War, UAS were also born under military requirements, specifically in the Middle East conflict. But once the technology is mature enough it starts to be used in civil and scientific missions.

The recent developments on increasingly smaller sensors, both for aircrafts and satellites, are directly useful for unmanned platforms. Most sensors are imaging technologies that include conventional cameras, infra-red/thermal cameras, multi spectrum cameras, radiometers, Doppler radar and synthetic aperture radar. Small and light cost-effective conventional and infra-red cameras exists today in the COTS market than can be included right away in a UAS. The rest of the sensor technologies are still too expensive or too heavy to be onboard a UAS, but increasing expected demands will accelerate their miniaturization. Also, new image processing techniques have been developed and now they can be embedded in small and cheap hardware boards and obtain end-users information in almost real time.

The introduction of this new type of air vehicle may be seen just as a new facility for remote sensing applications. But the capacities and costs of UAS compared against those of the other aerial vehicles may open new challenges and create new applications. In particular the three specific benefits of the introduction of UAS are: quality, opportunity and cost.

- Quality: It is clear that the quality of the information is directly related to the altitude at which sensors operate. The minimum orbit altitude for a satellite may range from $700 \mathrm{Km}$ (low altitude satellite like TERRA satellite from NASA) to $35000 \mathrm{Km}$ (geostationary orbits). The resolutions of sensor images taken from such altitudes range from $250 \mathrm{~m}$ to $1000 \mathrm{~m}$ (ie. the MODIS, Moderate-resolution Imaging Spectroradiometer, of the Terra satellite). With a similar sensor, the resolution archived from an aircraft that flies at $5000 \mathrm{~m}$ will improve dramatically.

- Opportunity: The huge number of Earth orbiting satellites (more than 500) gives a large imagery of Earth surface everyday. Depending on the payload/position of the satellite, the needed information for a given physical phenomena may not be available at the exact moment it is needed. For such applications aircrafts are a solution during day time. However, during night periods, dangerous situations, highly 
repetitive operations, or simply for low cost operation, the most practical, flexible and general solution is to use UAS for remote sensing.

- Cost: The use of satellite data has, in general, a subscription fee. End users contracts satellite services and thus, the global cost of the satellite system is assumed distributed between users and governments. In the case of aircrafts, the cost of a single operation may reach $5000 \$$ per hour. The payment of such rates may be worthwhile if the data in on time and of good quality. It is evaluated that the operational cost of a UAS may be smaller due to the saved cost of the pilot and the improved efficiency of the platform.

But today's market has not a generic UAS yet. Needs are solved with specific developments that incorporates the specific systems and specific mission programming. For this reason UAS costs are still higher that traditional airplanes. This paper proposes a generic UAS solution that can be applied to a large number of civil missions like observation and control of electrical lines, agricultural applications, traffic control or detection of fish banks, with a minimum cost of engineering. Developing an open-architecture avionics package specifically designed for UAS will alleviate costs by reducing many new developments to a simple parameterization.

UAS have the same limitations as most embedded systems: limited physical space, limited power resources, increasing computation requirements, complexity of the applications, time to market requirements, etc. In the competitive civil market, the same platform should be able to implement a large variety of missions and operate with many types of payload. Little reconfiguration effort and overhead should be allowed if the system has to be economically viable. For this reason we believe that the effective application of UAS in civil operations requires new systems that provide specific support to automatically control the actual missions to be carried out by the UAS.

\section{USAL Architecture for the Integration of UAS Missions}

This section describes the architecture we propose for executing UAS civil missions: a distributed embedded system that will be on board the aircraft and that will operate as a payload/mission controller. Over the different distributed elements of the system we will deploy software components, called services, which will implement the required functionalities. These services cooperate for the accomplishment of the UAS mission.

\section{III.A. Overview of the USAL Architecture}

The UAS System Abstraction Layer (USAL) is the set of available services running on top of the UAS system architecture to give support to most types of remote sensing UAS missions. ${ }^{6}$ USAL can be compared to an operating system. Computers have hardware devices used for input/output operations. Every device has its own particularities and the OS offers an abstraction layer to access such devices in a uniform way. Basically, it publishes an Application Program Interface (API) which provides end-users with efficient and secure access to all hardware elements. The USAL considers sensors and in general all payload as hardware devices of a computer. The USAL is a software abstraction layer that gives facilities to end-users programs to access the UAS payload. The USAL also provides many other useful features designed to simplify the complexity of developing the UAS application.

The USAL uses and also offers four communication primitives offered by an underlying service-oriented middleware: Remote Invocations, Variables, Events and File Transfers. The Remote Invocations are like the classical function calls and are used basically for initiation and finalization. Then the USAL includes as part of its API some standardized Variables and Events, common for all devices of the same type. Finally the capability of file-stream transfers are an alternative to Variables in case of sensors with large data acquisition capacities.

On top of this middleware, we have defined a collection of reusable services that comprises a minimum common set of elements that are needed in most UAS missions. A number of specific services have been identified as "a must" in any real life application of UAS (see Figure 2). The idea is to provide an abstraction layer that allows the mission developer to reuse these components and that provides guiding directives on how the services should interchange avionics information with each other. The available services cover an important part of the generic functionalities present in many missions. Therefore, to adapt our aircraft for a new mission it will be enough to reconfigure the services deployed in the UAS boards. 


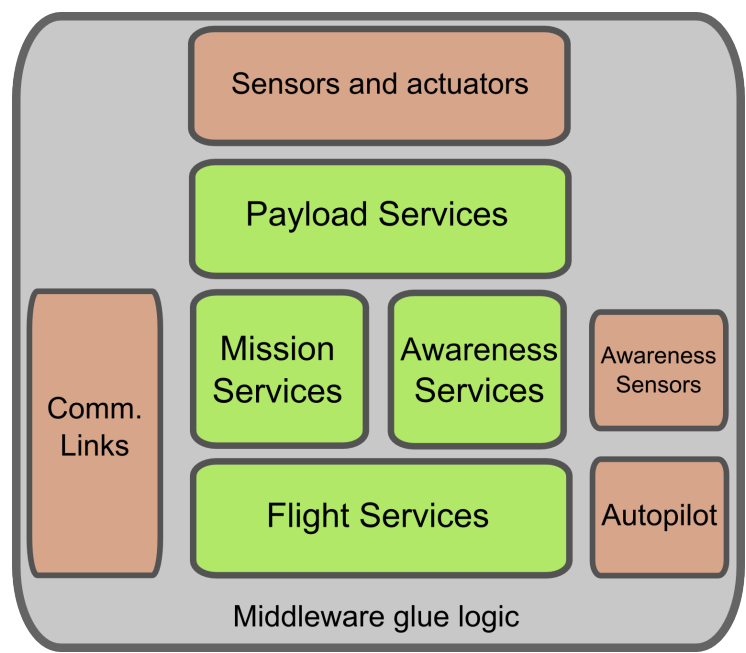

Figure 2. USAL Architecture Global View

\section{III.B. Service-oriented middleware}

Service oriented architectures (SOA) are getting common in several domains, for example Web Services in the Internet world ${ }^{7}$ and UPnP in the home automation area. The idea of these architectures is to increment the interoperability, flexibility and extensibility of the designed system and their individual components by using loosely coupled components.

SOA achieves loose coupling among interacting components by employing two architectural constraints. First, a small set of simple and ubiquitous interfaces to all participant components with only generic semantics encoded in them. Second, each interface can send on request descriptive messages explaining its operation and its capabilities. These messages define the structure and semantics of the services provided. These constraints are inspired significantly by object oriented programming, which strongly suggests that you should bind data and its processing together.

Our system, then, consists of a network of cooperating services, which implement the logic of the application and an integrating middleware layer that abstracts the execution environment and implements common functionalities and communication channels. ${ }^{8}$ In our network centric vision, when some service needs functionality not provided by itself, it asks the middleware for the required service. If another component of the system has this capability, its location will be provided and finally the client component will be able to consume the service using the common interface of the provider component.

This system composed of reusable services will be deployed over a number of low-cost distributed computing devices inside the UAS and connected by a local network. Our vision of the global system not only comprises the hardware onboard a single UAS, but also the ground control station, other mobile sensing devices and other UAS operating in the same network. In this view, the services are semantic units that behave as producers of data and as consumers of data coming from other services. The localization of the other services is not important because the middleware manages their discovery. The middleware also handles all the transfer chores: message addressing, data marshaling and demarshalling, delivery, flow control, retries, etc. Any service can be a publisher, subscriber, or both simultaneously. This publish-subscribe model virtually eliminates complex network programming for distributed applications.

The middleware allows the services to interact using different communication primitives: variables and events for periodic and urgent data transmissions, remote invocation for executing operations in other services and efficient multicast transmission of continuous media (files) to several services. Additional efforts has been placed in some specifics of UAS missions, in special the inter operation with unreliable and high-latency point-to-point networks when managing communications between UAS and ground control operators. 


\section{Advanced USAL Services}

Section III offers a general view of the USAL architecture and the underlying middeware technologies that allow the service oriented paradigm. In this section we will detail those services that are included by default into the USAL in order to facilitate the development of UAS applications. The existence of an open-architecture avionics package specifically designed for UAS may alleviate the developments costs by reducing them to a simple parameterization. The design of this open-architecture avionics system starts with the definition of its requirements. These requirements are defined by the type of UAS (mini or tactical UAS in our case) and the mission objectives.

\section{IV.A. USAL Services types}

Even though the USAL is composed of a large set of available services, not all of them have to be present in every UAS or in any mission. Only those services required for a given configuration/mission should be present and/or activated in the UAS. Available services have been classified in four categories according to the requirements that have been identified.

The principal element is the UAS autopilot. USAL considers the autopilot as a co-processor; it provides the system with a specific set of primitives that control the flight in the short term. The autopilot operation is supervised by a Flight Manager that abstracts users from autopilot peculiarities and offers flight plan specifications beyond classical way point navigation, thus improving operational capabilities. Additional services help improving the security and reliability of the operation. The services in charge of the flying capabilities of the UAS are named Flight Services.

The next relevant system is the computing system that should orchestrate the overall mission. This system may be joined by specific to mission additional systems like image processing hardware accelerators, etc. Storage and communication management should also be included by default. This set of standard plus user-defined services that control the mission intelligence are named Mission Services.

Payload includes all those other systems carried on board the UAS. The list of UAS hardware elements is completed with devices with less intelligence but with input/output capabilities. We divide them in data acquisition systems (or input devices) and actuators (or output devices). Input devices can be flight sensors (GPS, IMU, Anemometers) and earth/atmosphere observation sensors (visual, infra-red and radiometric cameras, chemical and temperature sensors, radars, etc.) Output devices are few or even do not exist in UAS civil missions because of the weight limitations: flares, parachutes or loom shuttles are examples of UAS actuators. Services controlling these devices are named Payload Services.

Successful integration of UAS in non-segregated aerospace will require a number of features to be included in the UAS architecture. Interaction with cooperative aircrafts through transponders, TCAS or ADS systems; and detection of non-cooperative aircrafts through visual sensors, should be implemented and the UAS must inform the pilot in command or automatically react following the operational flight rules for UAS that are currently being developed. ${ }^{9}$ However, for certain cases, e.g. flying in segregated airspace, such services may not be necessary. Services that manage the interaction of the UAS with the surrounding airspace users, controllers or conditions are named Awareness Services.

The proposed USAL architecture abstracts all these hardware components as services. To summarize, the USAL services are divided in the same three types we divided the hardware elements: Flight Services, Mission Services, Awareness Services and Payload Services.

- Flight Services are those in charge of basic UAS operation flight. This includes the autopilot management, the basic flight monitoring from end-users, the flight contingency management, the awareness services and all services in charge of the safe operation of the UAS with respect terrain avoidance and integration with shared airspace.

- Mission Services are those in charge of developing the actual UAS mission, controlling the payload and the area of surveillance, processing or saving the earth observation information and showing it to the end users.

- Payload Services are lower level services, not necessarily available to the end-users. They are like device-driver, this is, the facility services that abstract the details to access to the input, output and communication devices. 
Flight Services Category

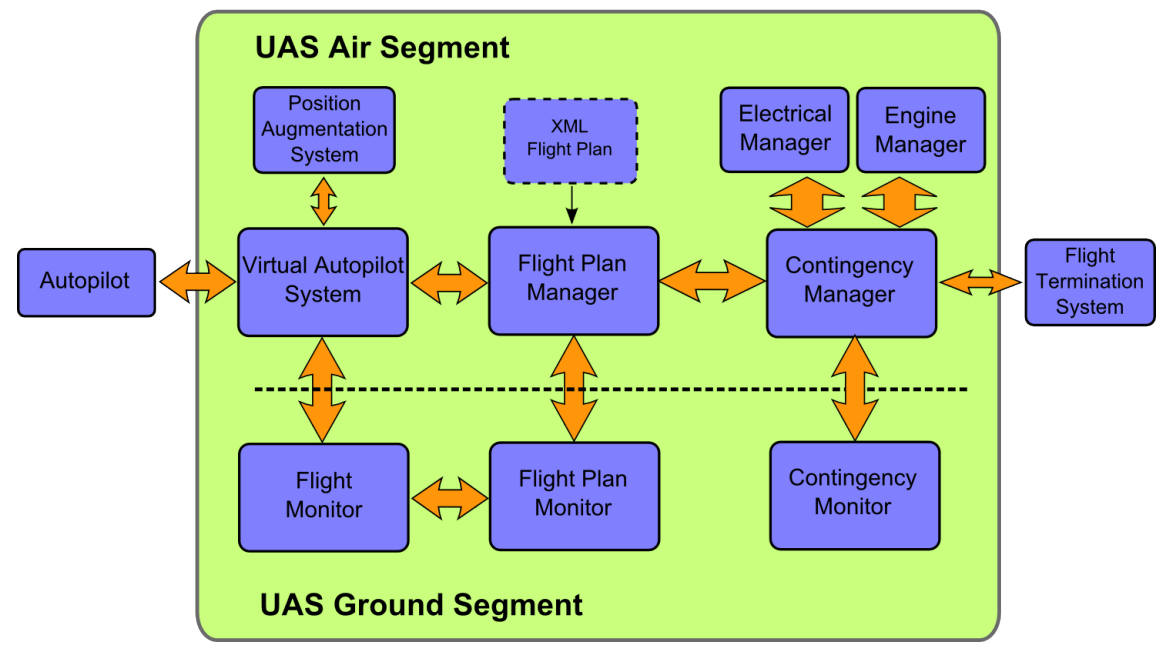

Figure 3. Overview of the Flight Services category

\section{IV.B. Flight Services}

Many autopilot manufacturers are available in the commercial market for tactical UAS with a wide variety of selected sensors, sizes, control algorithms and operational capabilities. However, selecting the right autopilot to be integrated in a given UAS is a complex task because none of them is mutually compatible. Moving from one autopilot to another may imply redesigning from scratch all the remaining avionics in the UAS. Current commercial UAS autopilots also have two clearly identified drawbacks that limit their effective integration with the mission and payload control inside the UAS:

- The complexity of exploiting on-board the autopilot telemetry by other applications is complex and autopilot dependent. Autopilots telemetry is typically designed just to keep the UAS state and position under control and not to be used by third party applications.

- The flight plan definition available in most autopilots is just a collection of waypoints statically defined or hand-manipulated by the UAS operator. However, no possible interaction exists between the flightplan and the actual mission and payload operated by the UAS.

Flight services are a set of USAL applications designed to properly link the selected UAS autopilot with the rest of the UAS avionics, ${ }^{10}$ namely the Virtual Autopilot Service, the Flight Manager Service, the Contingency Service, the Flight Monitor Service, etc. (see Figure 3):

- The Virtual Autopilot Service (VAS) is system that on one side interacts with the selected autopilot and is adapted to its peculiarities. VAS abstracts the implementation details from actual autopilot users. From the mission/payload subsystems point of view, VAS is a service provider that offers a number of standardized information flows independent of the actual autopilot being used.

- The Flight Plan Manager (FPM) is a service designed to implement much richer flight-plan capabilities on top of the available autopilot capabilities. The FPM offers an almost unlimited number of waypoints, waypoint grouping, structured flight-plan phases with built-in emergency alternatives, mission oriented legs with a high semantic level like repetitions, parameterized scans, etc. These legs can be modified by other services in the USAL by changing the configuration parameters without having to redesign the actual flight-plan; thus allowing the easy cooperation between the autopilot and the UAS mission.

- The Contingency Management services are a set of services designed to monitor critical parameters of the operation (like battery live, fuel, flight time, system status, etc.). In case contingencies are detected, actions will be taken in order to preserve the security and integrity of the UAS: from flight termination, mission abort or system re-cycle. 


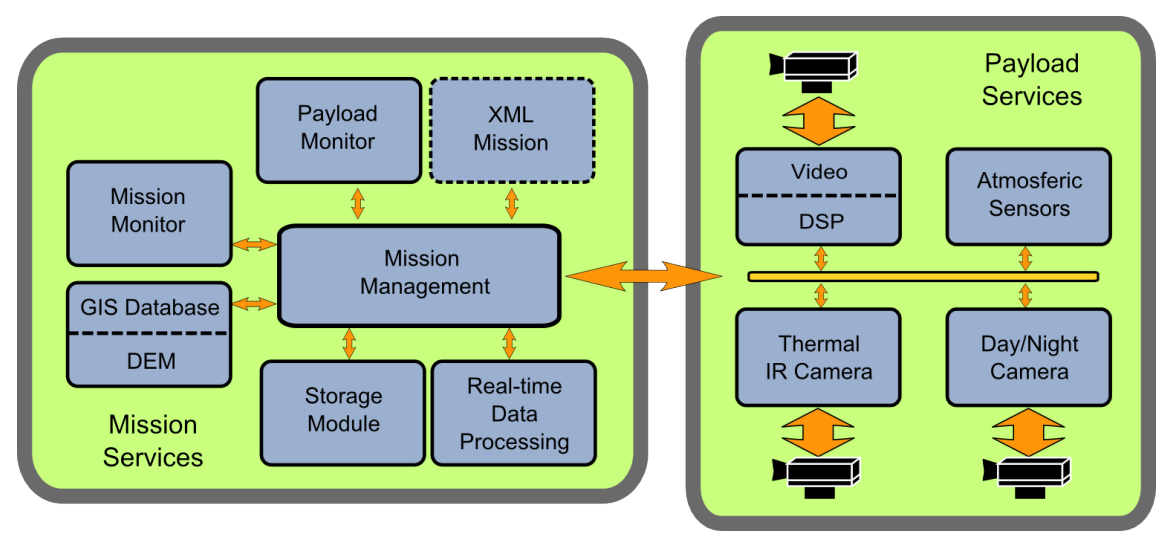

Figure 4. Overview of the Mission and Payload Services category

- The Electrical and Engine Management services are a set of services designed gather data on the operation of the UAS electrical system and the propulsion system. Such information is relayed to the Contingency Manager to take the appropriate decisions.

- The Flight Termination System is a system outside the USAL architecture, and it is in charge to deploy a parachute system in case the Contingency Manager requires it; also the parachute may be deployed in case a major USAL failure.

\section{IV.C. Mission Services}

From the end-users point of view, earth observation is the main target of a UAS flight. For this reason the user selects a geographic area that constitutes the initial objective of the observation. Also, the user must define the input sensors to activate and the conditions for missions updates. The DO-304 RTCA ${ }^{11}$ describes 12 scenarios, selected from a list of 70 as representative of UAS missions. Scenarios are representative of different types of airframes and navigation conditions. Regarding their navigation procedures, we have classified them in three types, with increasing complexity:

- Static Area Surveillance. The navigation pattern over the surveillance area is given before take-off. This navigation is the base of the following RTCA scenarios: Communicator Repeater (Scenario 7), Courier (Scenario 8), Border Surveillance (Scenario 16), Coastal Border Control (Scenario 26), High Altitude Communication Relay (Scenario 40).

- Target Discovering. The UAS system has a recognition service that can detect on-the-fly some objects or behaviors in the static area of surveillance. This navigation is the base of the following RTCA scenarios: Perimeter Defense (Scenario 3), OAV Police Operation(Scenario 10), Mass Casualty Analysis (Scenario 29). Although in some scenarios the mission continues after target discovering, the mission intelligence is relegated to ground operators decision and control.

- Dynamic Target Tracking. The most advanced missions are those that assume intelligence Mission Services, with the capacity of redefine the surveillance area with no human intervention. Hurricane Chase (Scenario 2), Coast Guard Reconnaissance and Surveillance (Scenario 37) are scenarios where this situation is given at some level.

In general, the most complex missions include the simplest missions as part of its objective. In example, in order to track a moving object, its previous discovering is needed, which is based on an area surveillance mission. For the Static Area Surveillance the only condition needed is the end-of-mission condition, but for the other two the end-users has to give the condition for Target Recognition.

The USAL offers a number of predefined services to implement a wide range of missions, namely the Mission Manager, the Real-Time Data Processing, the Storage, the Scheduled Communications the GIS/DEM Database and Mission Monitor (see Figure 4). These services can be adapted to requirements by means of parameters (e.g. specific flight plan, sensors to be activated, information flows, etc), by adding specific 


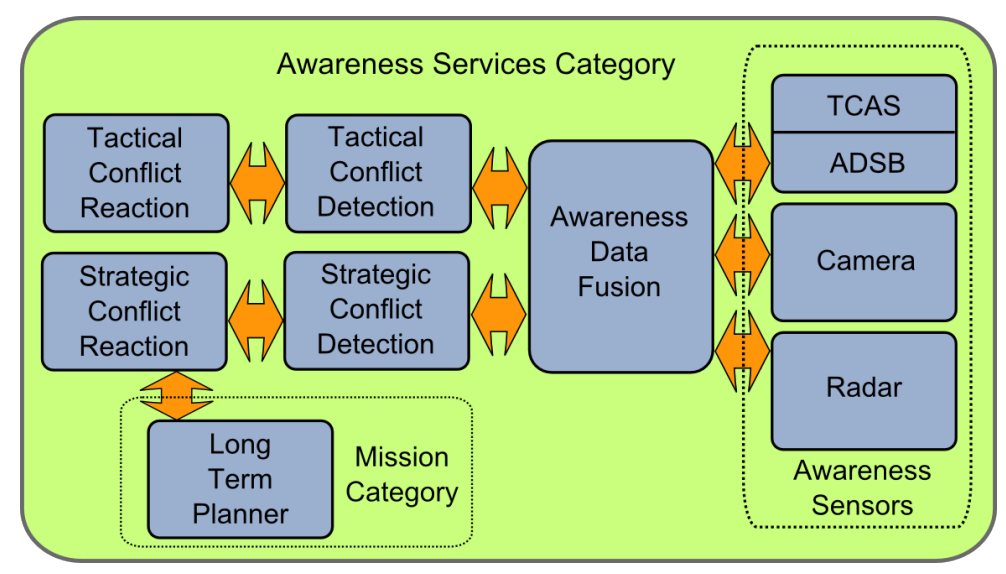

Figure 5. Overview of the Awareness Services category

software code to be executed or by adding specific user defined services. Next we describe in more detail some of them:

- The Mission Manager (MMA) is the orchestra director of the USAL services. This service supervises the flight services and the payload services; as well as the coordination of the overall operation. The MMA executes a user defined automata with attached actions (i.e. service activations) at each state or transition. Actions can be predefined built-in operations or specific pieces of user code. In particular the MMA is capable of modifying the actual flight plan by redefining its parameters or by defining new stages or legs.

- The Real-Time Data Processing (RDP) gives the intelligence for complex missions. The RDP offers predefined image processing operations (accelerated by FPGA hardware if available) that should allow the MMS to take dynamic decisions according to the actual acquired information.

- The Mission Monitor (MMO) shows to end-users a human friendly useful information about the mission. For example, during a wildland fire monitoring mission, it may present the current state of the fire front over a map. The MMO is basically executed on the ground and should be highly parametrized to fit the specific requirements of each mission.

- The Payload Monitor (PM) gathers information on the health status of all payload registered in the USAL. This information may be sent to the Contingency Manager in order to detect mission contingencies and to an equivalent payload monitor on the ground.

- A GIS database and a DEM database are also available on-board in order to offer geografical information to other services that may need it.

\section{IV.D. Awareness Services}

A UAS is a highly instrumented aircraft and has no pilot on board. With these conditionings the more suitable flight rules for a UAS are IFR, however for remote sensing missions the advantages of UAS systems is precisely its capacity for flighting at any altitude, where VFR aircrafts are found. UAS must relay on its instrumentation equipment to properly inform the pilot in command on the ground or substitute the pilot capacities in VFR conditions. The awareness services are responsible of such functionalities. Flight Services are in charge of the aircraft management in normal conditions while the Awareness Services are in charge of monitoring surroundings conditions and overtake aircraft management in critical conditions. In this case mission services come to a second priority, until flight conditions become again normal. The lists of flight services are (see Figure 5):

- The Awareness data fusion (ADF) is a service designed to collect all available data about air vehicles surrounding our UAS, terrain and meteorological conditions. All this information can be optained either by on board sensors or even through an external provider. 
- The Tactical/Strategic Conflict Detection service will analyze the fused information offered by the ADF in order to detect potential collision conflicts with objects/terrain/bad climate. Depending on the type of conflict, different types of reaction procedures will be activated. While reaction is executed it will keep monitoring than the conflict is really being avoided.

- The Tactical/Strategic Reaction services, will implement avoidance procedures according to the severity of the conflict. Tactical reaction is designed in such a way it can overtake the Flight Plan Manager in order to execute a radical avoidance maneuver. Once completed, the FPM will regain control. An strategic reaction will command the FPM to slightly modify its selected flight plan trying to avoid the conflict but at the same time retaining the original mission requested by the Mission Manager.

- A set of dedicated awareness sensors will acquire the necessary information in order to learn about conflicting collaborative and non-collaborative aircrafts.

\section{IV.E. Payload Services}

Payload services are defined for low level devices, mainly raw data acquisition sensors that need to be processed before being used in real-time or stored for post-mission analysis. The complete list of services is directly related to available sensors, and except for most classical cameras they need to be created or adapted by the end user. However, USAL offers pre-build skeletons that should be easily adapted for most common devices.

\section{UAS Remote Fire Monitoring Architecture}

This work introduces a flexible and reusable architecture designed to facilitate the development of remote sensing applications. Based on it, we are developing a helicopter system, called Sky-Eye, devoted to the detection, control and analysis of wild land forest fires in the Mediterranean area. The general architecture designed for wildfire monitoring.

\section{V.A. Background}

Wildfire monitoring is becoming one of the preeminent remote sensing applications due to its environmental, economical and social implications. Between $200.000--600.000 h a$ are burnt every year in Europe; $80 \%$ corresponds to Mediterranean forests with high environmental value.

This impact is accentuated in densely populated areas like Southern California. In October 2003, 14 fires in 6 counties consumed over 750,000 acres of wildlands, destroying 3,338 residential structures, 33 commercial properties, 1,072 outbuildings, and killing 26 people. The conflagration caused the greatest mobilization of firefighting resources in Californias history with costs totaling 3 to 5 billions. Again, in October 2007 wildfires spread across Southern California with nine people killed, 85 injured, 1,500 homes destroyed and 500,000 acres burned from Santa Barbara to the U.S.Mexico border.

For the first time during the October 2007 fires a UAS participated in real-time wildfire monitoring activities. NASA's Ikhana UAS flew over the major Southern California wildfires from Oct. 24 until Oct. 28 , capturing thermal imagery to aid firefighters. NASA's Ikhana unmanned aircraft system flew a number of missions over several of the major Southern California wildfires from Oct. 24 until Oct. 28, capturing thermal-infrared imagery to aid firefighters. Ikhana, a General Atomics' Predator B adapted for civil science and technology research missions, flied long-endurance sorties lasting nine to 10 hours each from its base at NASA's Dryden Flight Research Center. ${ }^{12}$

Up to now, satellites have been the primary source for large area thermal imaging. Satellites like the NASAs Terra and Aqua acquire information from its Advanced Spaceborne Thermal Emission and Reflection Radiometer (the MODIS system), offer almost real-time active fire maps twice a day with automatic detection of thermal anomalies. ${ }^{13}$ However, UAS can complement this information with much detailed imagery at any time of the day or night. Ikhana is the first UAS that experimentally executes this mission on real fires.

\section{V.B. Motivations to UAS-based monitoring}

Decisions on the forest fire management should be basically taken on the actual fire itself. However, several factors restrict the capacity to obtain a global and precise situation by those responsible of taking the right 


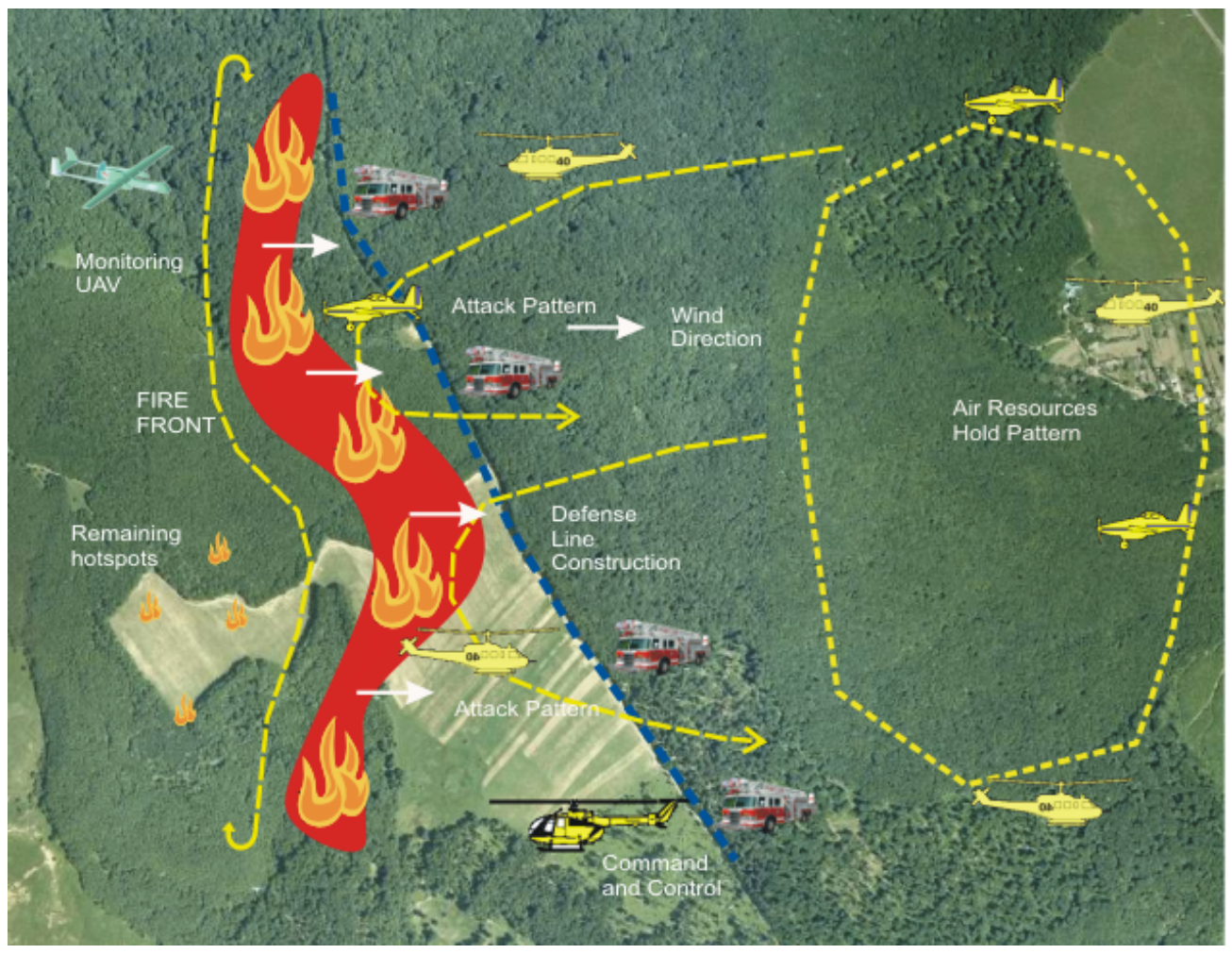

Figure 6. Classical air/ground operations for wild land fire contention.

decisions. Smoke interferes with the ability to acquire coherent data about the actual situation. If it is necessary to obtain a global perfective of the fire, the coordinator should over-fly the area, thus leaving its control center temporally. Using manned airplanes for forest-fire control cannot be used during the night or in presence of dense smoke, flying time is quite limited, available sensors on board traditional airplanes are almost non-existent, etc. Also, dangerous situations for the fire extinguishing personnel working on the ground may develop very rapidly.

Current technology used to collect information on the operational situation on the ground prevents that the fire fighter coordinator to choose the right decisions on time. A UAS platform capable of over flying the area of a forest fire for long periods of time and with capacity of operating from non-prepared terrains would be an extremely valuable information gathering asset, overcoming some of the aforementioned limitations. The added values of using an UAS in any civil task scenario are:

- May be used in dangerous flight conditions, as nocturnal flights or with low visibility.

- Payload can acquire coherent data even with bad weather or environmental conditions.

- May increase users ubiquitous information and help decision-makers.

- May prevent dangerous situations for ground brigades.

- May provide rely links between the ground teams, especially in mountain or non-covered areas.

The requirements for the design of such UAS system are:

- The cost of the system and its operation should be much less than the cost of the conventional means.

- The data should be acquired continuously, during day and night, or in adverse meteorological conditions.

- The information should be sent periodically to a ground station, analyzed and offered in real time.

- The system must avoid any dangerous situation for the life of people: either pilots or ground teams. 


\section{V.C. System architecture}

The proposed UAS system will be composed of five main components. Each component will work collaboratively to constitute a platform of high added value to the people in charge of surveillance and to help to make their task more accurately. The components of the proposed solution are:

- UAS, sensor platforms and embedded control system.

- Mobile ground control station.

- Data processing and storage center.

- Ground brigade information terminal

- Air/ground communication infrastructure.

Figure 6 depicts the general application scenario: a wildland forest with active fire fronts being contained by ground brigades while aerial resources attack the fire front in a circuit-based pattern. A tactical UAS provides continuous day/night monitoring to the command center and relies information to ground brigades. However, the integration of a tactical UAS in this scenario is a complex and unsolved problem due to the highly dynamic and complex behavior off all aerial vehicles operating over the fire area.

\section{V.C.1. UAS, sensor platforms and embedded control}

The UAS platform and sensor set is selected according to the targeted "Mediterranean" area and cost objectives. A medium altitude tactic UAS is preferred for cost availability. Basically two cameras need to be installed on-board: a high definition visual camera and a thermal camera configured for temperature ranges according to the precise mission to be implemented. More details on missions are offered in SectionV.D. Multi-spectrum thermal sensors are also desirable but its availability, cost and possible integration in a tactic UAS is uncertain.

In addition to the autopilot/GPS-navigation subsystem, the avionics in the UAS should include hardware support to execute all USAL services required to properly manage the remote sensing mission: sensor management, data storage, communications, image processing, flight-plan management and mission management. As previously described it is assumed that all those processors are internally networked currently through an Ethernet switch or router.

All data acquired by the sensor package will be internally stored and partially processed in real-time on board the UAS. Selected telemetry and sensor information will be scheduled to be transferred to the ground for more detailed analysis. All data can be later on transferred to the ground when the UAS gets back on the ground or during flying periods in which no other high-priority data is scheduled to be transferred.

\section{V.C.2. Mobile ground control station}

The ground teams working on the surveillance area should have equipment available to directly control the UAS operation in real time. Additionally, immediate "fire"-related information should be made available to this control station so that fire operations coordinators can use it regardless of further distribution of the information to other control centers.

This is the goal of the Mobile ground control station (MGCS) The objective is to be able to control the system close to the area of actuation, relaying on line-of-sight communications (due to the cost and complexity of satellite communications). UAS status, actual state of the mission operation and the initially obtained surveillance data will be received there, although other data sources in addition to the UAS itself may be available. The MGCS will offer a basic graphical GIS interface for quasi real-time management of data and for mission re-tasking according to the fire evolution. Hot-spot management as well as firefront detection and evolution could be completed here including a true orto-normalization of the overall information.

The development of the mission should be followed at any moment and modified when it is opportune from MGCS. 


\section{V.C.3. Ground Brigade Information Terminal}

A set of PDA clients carried by fire brigades will be integrated into a wireless network formed by the UAS and the MGCS. These PDAs will receive and present information from the MGCS (which could include messages, pictures, videos, maps, alarms, commands. They can also transmit data from a set of attached sensors to the MGCS, which includes: GPS position of the brigade, pictures, video, messages, ambient temperature and humidity, etc. The position of all information terminals is integrated in the mission interfaces offered by the MGCS to the fire operations coordinator.

\section{V.C.4. Data Processing and Storage Center}

The MGCS is conceptually designed to control a single UAS or at most a few number of units to guarantee 24 hours 7 days a week coverage. However, in case multiple UAS teams are available and operate on different fires they should be coordinated from a Data Processing and Storage Center (DPSC). In addition to the coordinated operation of multiple UAS teams it will be necessary to have a system in which store all mission data for high-level supervision and decision making. More powerful analysis tools may be available at this processing center and the resulting products can be re-sent back to the MGCS. The DPSC also plays a role as a mission data-base system to high volume information storage and mission replay for incident analysis/evaluation.

\section{V.C.5. Air/ground communication infrastructure}

The availability of a reliable communication connection between the UAS and the MGCS, and eventually with the Brigade Information Terminals is critical. However, the existence of a mixture of communication connections should not negatively interfere with the development of the various mission services involved in the application. The middleware will properly manage these connections according to the priority of the information to exchange, the available bandwidth, and the cost of each connection.

The wildfire monitoring system will initially rely on a variety of commercially communication subsystems; namely the embedded radio modem in the selected autopilot, a wifi/wimax module for high bandwidth data transmission, a GPRS/UMTS OEM system and a spread spectrum commercial radio modem. Eventually, a commercial satellite link can be added depending on the budget availability.

Transmission rules will limit full resolution image exchange to the wifi/wimax link. Telemetry and UAS control will have the highest priority among all other information flows. Mission control will manage the acquired sensor information, those tagged as highly relevant will be scheduled for transmission either in full format or in reduced resolution. In case the available bandwidth is not sufficient, even for reduced resolution images, images will be analyzed in order to extract meta information like hot-spot location and/or position of fire fronts. Then, this meta-information can be exchanged even with the lowest bandwidth data links.

\section{V.D. Tactical Application Scheme}

The general architecture designed for wildfire monitoring described in the previous sections can be specially tailored for two relevant objectives within the particular wildland Mediterranean scenario:

- Day/night fire front evolution,

- Post-fire hot-spot detection.

\section{V.D.1. Day/night fire front evolution}

The objective of this type of mission are surveillance flights during day and night to gather the information required by fire-fighters to conduct extinction operations in a timely and effective way. Such flight should not interfere with the operation of other aerial resources.

From a technical point of view the UAS should be capable of automatically identify the perimeter of the fire and follow it in successive scans. ${ }^{14,15}$ In addition to the imagery itself, the geographical delimitation of the perimeter will allow to identify two critical informations:

- The dynamic evolution of fire fronts including the speed at which each front evolves

- Detect ground brigades that may become trapped by fire fronts 
- Detect whether fire contention is effective

The detailed design of this type of mission is still under analysis and development with fire managers in order to clearly identify the "real" operative and integration requirements.

\section{V.D.2. Post-fire hot-spot detection}

Due to the current legal situation of UAS in Europe it is not possible to execute fire front evolution missions. The integration of the UAS with the rest of aerial resources is an unsolved problem, both technically and methodologically. However, the operation of UAS during certain very well identified phases of the extinction process in highly plausible. In addition to monitoring the fire front evolution, detection of remaining postfire hot-spots located at the perimeter of fire ${ }^{16}$ is the main UAS application that has been foreseen in the European Mediterranean area.

Just after a fire front is contained or even during the days following the fire extinction, the monitoring tasks have to be maintained because of the danger of fire reactivation. The cost of monitoring with ground teams or aerial means it is very expensive and consumes resources needed in other fronts or possibly concurrent fires. However, a UAS equipped with thermal cameras, can flight over the area and generate a map of hot spots with higher precision at a smaller cost. It is crucial in this application that the hot spots are reported immediately to avoid having ground brigades waiting for data for too long. Also, it is important to feedback the information in such a way it can be effectively consumed, not forcing ground brigades to walk around the forest without a clear operational scheme.

\section{Operation of the Tactical Hot-spot System}

This section will detail a solution proposal to implement a post-fire hot-spot detection system based on an helicopter UAS that is linked to a mission control on the ground (the Red-Eye system). The designed command and control systems software on board the helicopter and on the ground are almost identical. The main difference between them is the latency required to get the image information. On board the helicopter images can be reviewed as soon as acquired (although hot-spot detection will require certain computation time). On the ground, images can be seen under request and the latency of the transmission will be much longer and will depend on the distance from the helicopter to the ground control.

The operation of the command and control software is divided in five different phases, namely:

1. System configuration.

2. Definition of exploration areas.

3. Hot-spot exploration.

4. Hot-spot confirmation.

5. Ground squad task assignment.

These operational phases are supported by a mission monitoring tool depicted in Figure 7.

\section{VI.A. System configuration}

The system configuration screen allows configuring the system in order to define the preferred exploration altitudes and speeds according to the desired resolution in each phase of the operation (exploration/confirmation) according to the available combination of camera/optics. Information indicating the location of the ground control station, landing sites, identification of the available ground squads can be also specified. Finally, restricted areas can also be specified to indicate population areas not to be directly over flown and/or especially dangerous obstacles like towers or electric lines.

\section{VI.B. Definition of exploration areas}

The areas to be explored need to be specified by the operator by means of a closed polygon. Each polygon implies, at least, a separated exploration by the helicopter. The Sky Eye system automatically offers an exploration solution defined by a number of trapezoidal areas to be scanned through linear tracks over flown 

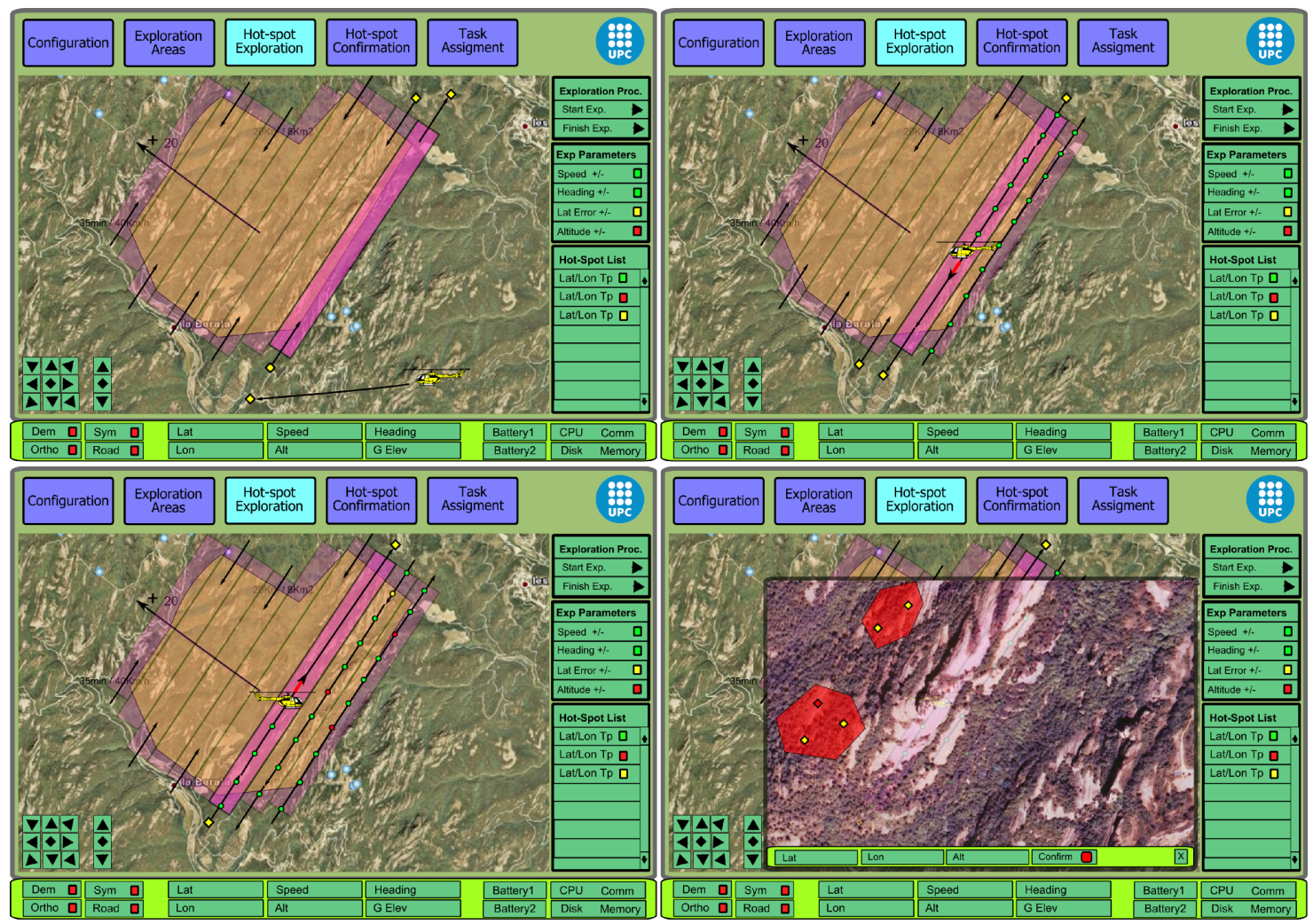

Figure 7. Overview of the user interface for the Red Eye hot-spot mission.

by the helicopter at the specified distance to the ground. The particular altitude to be flown in each pass will depend on the variations of the terrain elevation (available through a Digital Elevation Model of the area). Figure 7 shows different views of the application for two different exploration areas. The main direction of the terrain elevation is specified by an arrow annotated with the average terrain slope. High slopes will force perpendicular explorations to avoid flying into the ground, while small slopes can be ignored is the selected altitude guarantees than no collision is possible.

\section{VI.C. Hot-spot exploration}

Once the exploration scans have been properly defined, the scanning process itself can be started. The system automatically generates Initial Scan Points (ISP) and Final Scan Points (FSP) to guide the task of the pilot. The ISP allows the pilot to properly align the vehicle with the exploration track, while the FSP provides room for the turning maneuver and the re-alignment with the following exploration track.

During each exploration track the Red Eye cameras are automatically activated in order to gather images at the required frame rate (that will depend on the camera/optics, the selected altitude and the desired frame overlapping between consecutive frames). Each frame acquisition is indicated on the screen as a green dot. Frame acquisition stops once the FSP is surpassed and re-stars as soon as the helicopter gets into the next ISP.

While the exploration is being completed thermal images are being analyzed in order to determine if they have a significant thermal signature. In that case the corresponding dots may turn into red or yellow, depending on the intensity of the thermal signature. Additionally, the source of the hot spot is identified inside the image and then geo-referenced according to the information provided by the IMU and GPS at the time of the acquisition. The analyzed information is offered to the controller in order to confirm if it is a real hot-spot or maybe stray heat reflections from some element on the ground (rocks, human-made devices, etc.). Thermal images can be over imposed on top of high-definition images in order to facilitate the identification of the hot-spot (see Figure 8 for an experimental example of such superposition).

Figure 9 shows a simulated exploration track generated to analyze a given area. Once the area has been 


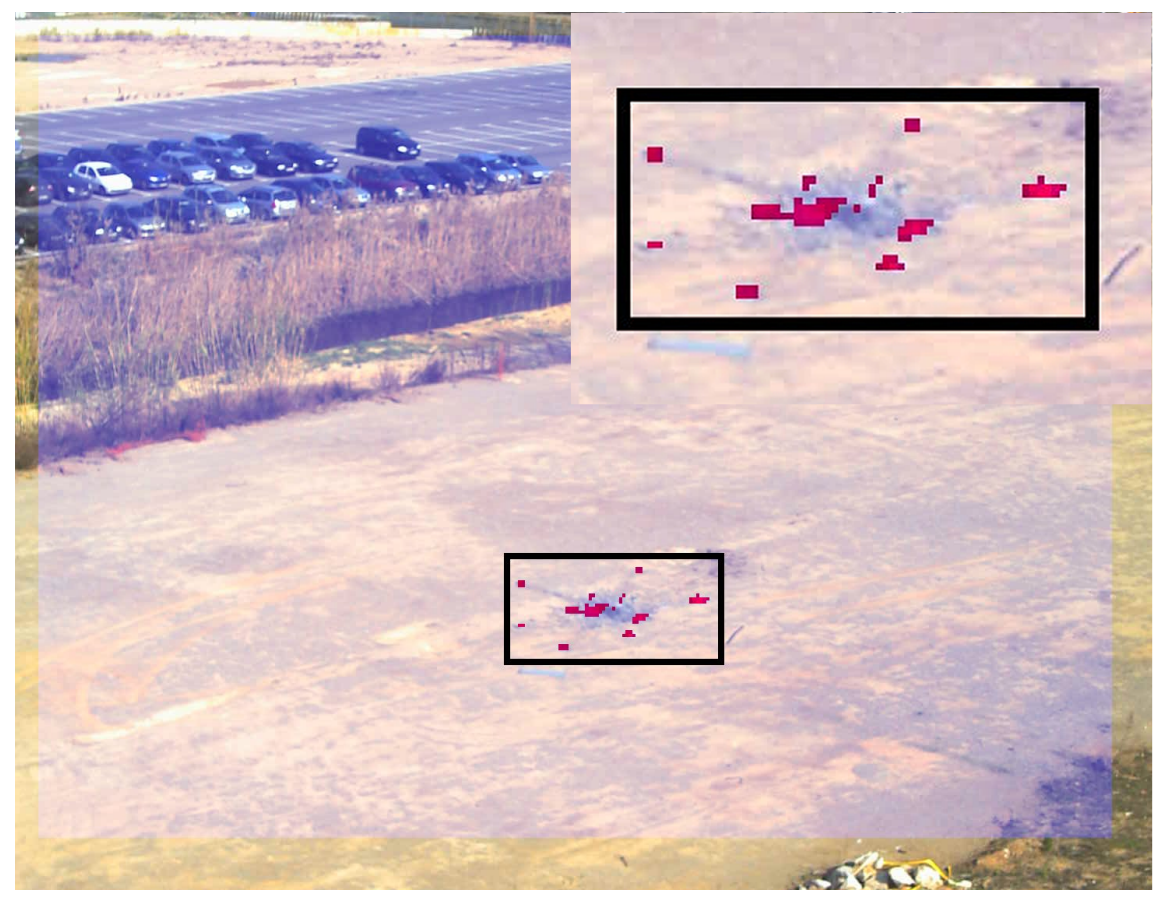

Figure 8. Example of hot-spot identification through thermal/visual image fusion.

completely analyzed and hot-spots detected (maybe some hold time is required until the acquired imagery has been fully processed), the exploration continues by overflying at lower level the hot-spot locations. Explorations are selected at users discretion and can be repeated if necessary and/or interleaved with the confirmation phase.

\section{VI.D. Hot-spot confirmation}

After an exploration is being completed some detected hot-spots may be still undecided. The Red Eye system offers support to design a trajectory that overflies all undecided hot-spots in order to analyze them at a lower altitude and/or during a hovering flight. Having a closer look on the potential hot-spot should help deciding its relevance.

Hot-spots are processed sequentially following the sequence suggested by the application. Confirmation sequences can be repeated after the ground squads have refreshed the area. In that way, detected hot-spots can be revisited to determine if the extinguishing task have been successful.

\section{VI.E. Ground squad task assignment}

Given the set of confirmed hot-spots, the controller can assign them to the available ground squads working on the area. Ground squads will receive all information available on the hot-spot like its position, thermal and visible images and any available detection information.

Once the squad has identified the hot spot and worked on it, they will communicate the controller through the application that the spot has been processed. During this process, the controller will be able to follow the progression of the teams on its screen.

\section{VI.F. Overview of the mission operation}

Figure 10 outlines the USAL services and the mission methodology being designed in order to complete the hot-spot mission. Currently, the starting point of the overall process is the determination of the risk area to be analyzed. This area is manually specified by the system operator, although it is being investigated a methodology to real-time identify post-fire perimeters combining thermal and visual information. 


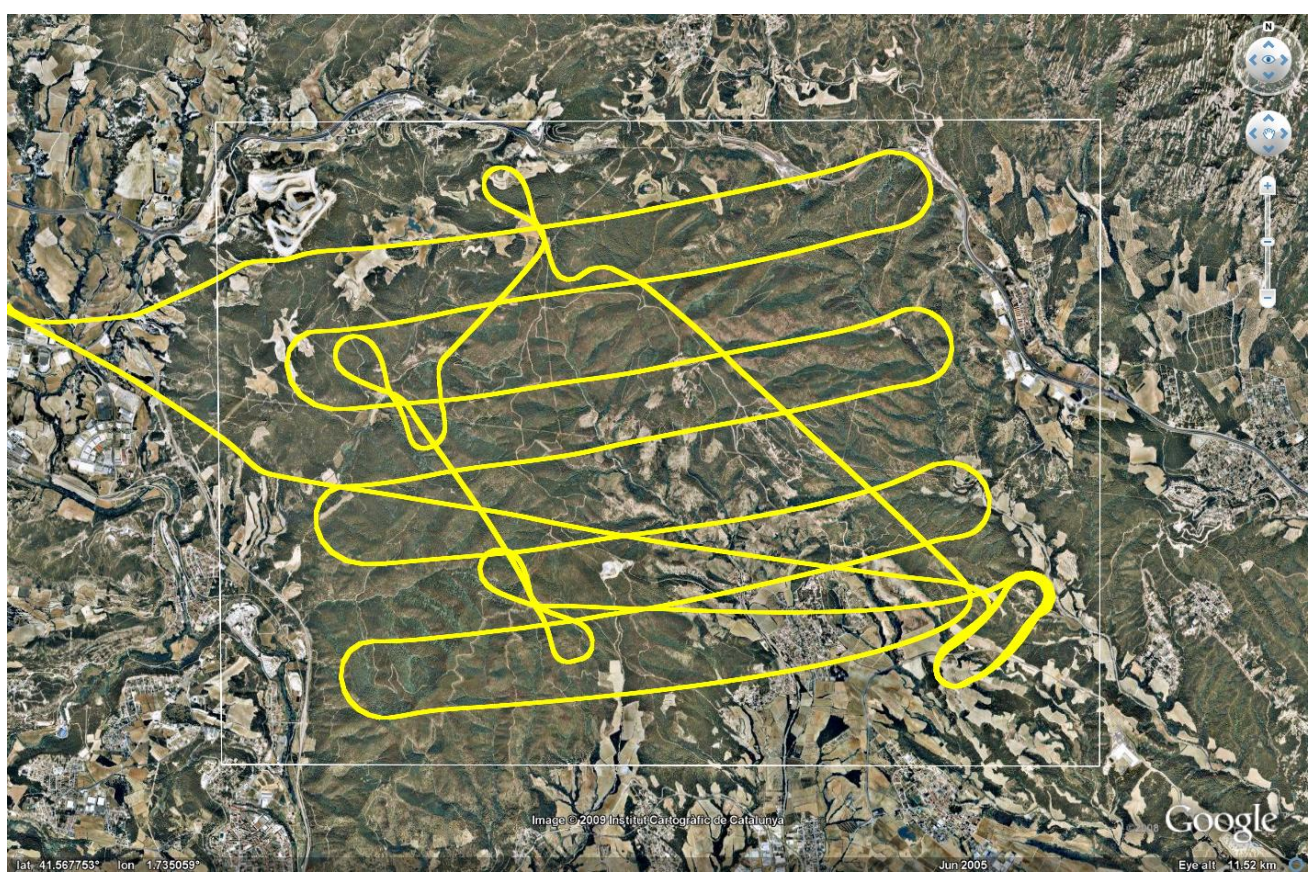

Figure 9. Automatic flight pattern executed by the FPM service according to the feedback provided by on-board payload.

Thermal images will be acquired following scan patterns defined by high-level parameters determined by the Mission Manager. Hot spots will be detected and stored in a selection table. Given that the same hot-spot can be detected in various images, a single image representative will be stored - the one with more thermal radiation detected in the hot spot. Note that an initial filter will be used in order to identify relevant images on-board the UAS. Those images that contain relevant thermal information will be analyzed in detail to identify the exact location of the hot-spots in the image and later geo-reference the image according to the instantaneous location and orientation of the vehicle, and the elevation model of the area under exploration.

After a block of consecutive scans has been executed a number of decisions are taken. First, to remove all those images not really referenced by any hot-spot. Then, to transmit to the Mission Monitor on the ground the list of hot spot coordinates through the highest priority communication link, while the thermal images with hot spots highlighted on them will be scheduled to be sent as soon as high bandwidth communication links are available. Together with the thermal information the corresponding high quality visual image will be also sent. The combination of both images can be analyzed by the fire coordinator in order to precisely locate the position of the hot-spots, even under the existence of location errors due to GPS error or alignment error of the on-board inertial sensors.

Finally, it is worth noting that even though only selected information is scheduled to be sent to the Mission Monitor on the ground, all other information will be stored on-board to be later downloaded for detailed analysis.

\section{Conclusions and Future Work}

UAS are becoming a new major platform that may help to improve classical remote sensing applications as well as creating new remote sensing paradigms. UAVs may eventually replace manned airplanes allowing much more cost-effective operations and will remain complementary to classical satellite applications. The class of mini/micro UAS will become available to many research institutions, universities, and private companies to develop their research or commercial applications. However, current technology offers solutions for most components in the UAS system except systems to support and automate the actual sensing mission.

This paper has introduce USAL, a service-oriented architecture designed to support the development of remote sensing applications. USAL offers a number of pre-defined services that can be easily parametrized to the specific needs of the application. Additional services can be introduced to incorporate new functionalities 


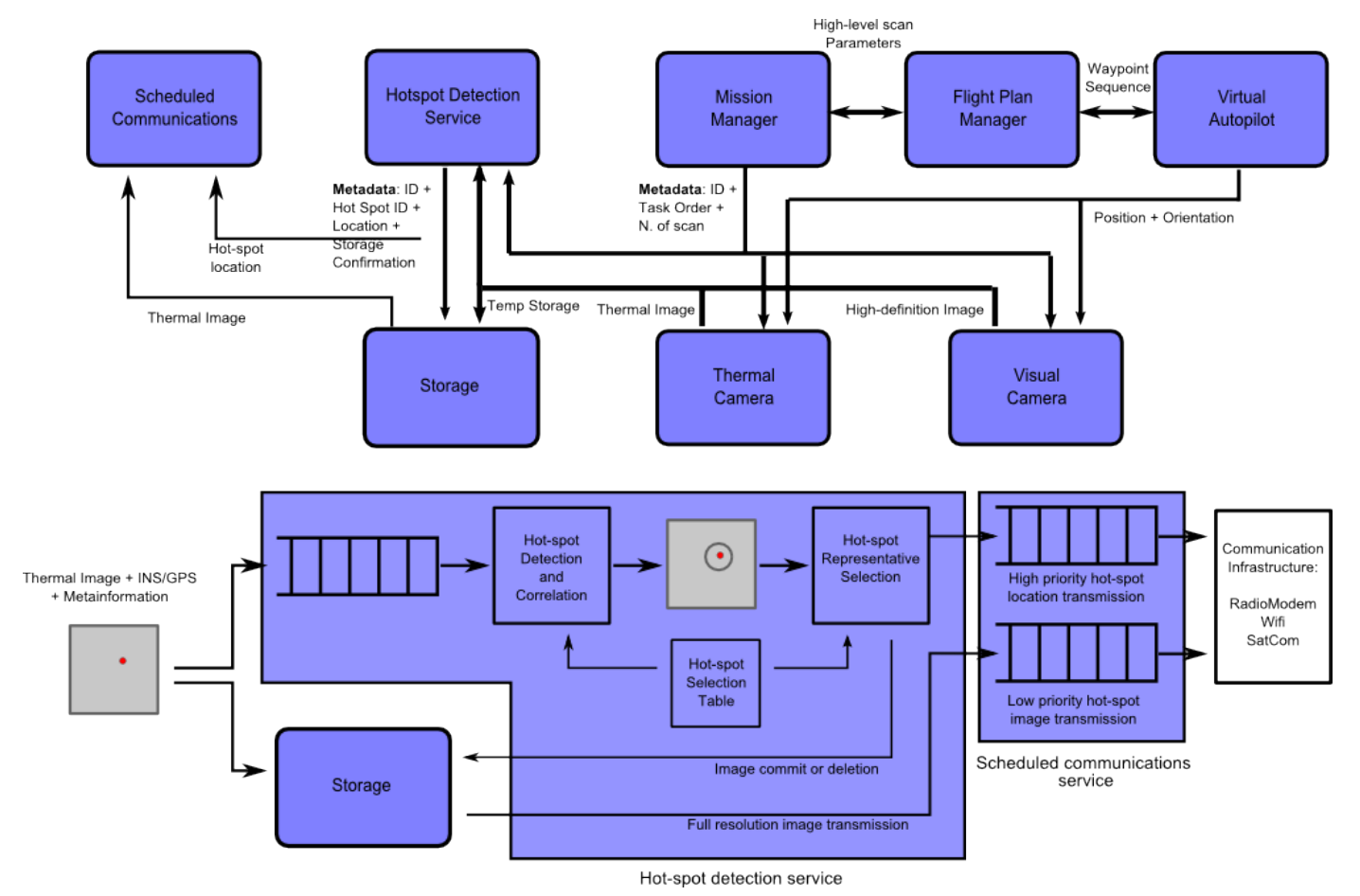

Figure 10. Outline of the USAL operation for the hot-spot UAS mission.

and at the same time reusing available services. A middleware designed to support the type of inter-service communications required by the USAL is also introduced. The design of a UAS system for the monitoring of wildland fires in the Mediterranean area, with special emphasis on the hot-spot monitoring application, is used as an application example of the USAL. Future research will address the development of the complete fire perimeter monitoring mission and the parameterizable ground-based mission monitoring stations.

\section{Acknowledgments}

This work has been partially funded by Ministry of Science and Education of Spain under contract CICYT TIN 2007-63927.

\section{References}

${ }^{1}$ Wegener, V. A. S. and Brass, J., "The UAV Western States Fire Mission: Concepts, Plans and Developmental Advancements," AIAA 3rd "Unmanned Unlimited" Technical Conference, Workshop and Exhibit, AIAA, Chicago, Illinois, 2004.

${ }^{2}$ Loegering, G., "Global Hawk - A New Tool for Airborne Remote Sensing," 1st UAV Conference, AIAA, Portsmouth, Virginia, 2002.

${ }^{3}$ MacDonald, A. E., "A Global Profiling System for Improved Weather and Climate Prediction," Bulletin of the American Meteorological Society, Vol. 86, No. 12, 2005, pp. 17471764.

${ }^{4}$ Nichols, J. D., "Firefly system concept," Proc. SPIE Vol. 1540, p. 202-206, Infrared Technology XVII, Bjorn F. Andresen; Marija S. Scholl; Irving J. Spiro; Eds., edited by B. F. Andresen, M. Scholl, and I. J. Spiro, Vol. 1540 of Presented at the Society of Photo-Optical Instrumentation Engineers (SPIE) Conference, Dec. 1991, pp. 202-206.

${ }^{5}$ Kaufman, Y., Justice, C., Flynn, L., Kendall, J., Prins, E., Giglio, L., Ward, D., Menzel, W., and Setzer, A., "Potential global fire monitoring from EOS-MODIS," Journal of Geophysical Research, Vol. 103, No. 32, 1992, pp. 215-238.

${ }^{6}$ Pastor, E., Lopez, J., and Royo, P., "UAV Payload and Mission Control Hardware/Software Architecture," IEEE Aerospace and Electronic Systems Magazine, Vol. 22, No. 6, 2007.

${ }^{7}$ W3C, "W3C Note: Web Services Architecture," http://www.w3c.org/TR/ws-arch.

${ }^{8}$ Lopez, J., Royo, P., Pastor, E., Barrado, C., and Santamaria, E., "A Middleware Architecture for Unmanned Aircraft Avionics," ACM/IFIP/USEUNIX 8th Int. Middleware Conference, NewPort, California, Nov. 2007.

9 "EUROCONTROL specifications for the use of military unmanned aerial vehicles as operational air traffic outside segregated airspace," European Organisation for the Safety of Air Navigation, 2006. 
${ }^{10}$ Santamaria, E., Royo, P., Lopez, J., Barrado, C., Pastor, E., and Prats, X., "Increasing UAV capabilities through autopilot and flight plan abstraction," Proceedings of the 26th Digital Avionics Systems Conference, Dallas, Texas, 2007.

${ }^{11}$ RTCA, "DO-304: Guidance Material and Considerations for Unmanned Aircraft Systems," March 2007.

${ }^{12}$ Ambrosia, V. and Schoenung, S., "The 24-Hour UAV Western States Fire Mission: Sensor and Intelligent Management Systems,"Infotech@Aerospace, AIAA, Arlington, Virginia, 2005.

${ }^{13}$ Giglio, L., Descloitres, J., Justice, C., and Kaufman, Y., "An enhanced contextual fire detection algorithm for MODIS," Photogrammetric Engineering and Remote Sensing, Vol. 87, 2003, pp. 273-282.

${ }^{14}$ Casbeer, D., Kingston, D., Beard, R., and McLain, T., "Cooperative forest fire surveillance using a team of small unmanned air vehicles," International Journal of Systems Science, Vol. 37, No. 6, 2006, pp. 351-360, Publisher: Taylor and Francis Ltd.

${ }^{15}$ Ononye, A., Vodacek, A., and Saber, E., "Automated extraction of fire line parameters from multispectral infrared images," Remote Sensing of Environment, Vol. 108, No. 2, 2007, pp. 179-188, also AIAA Paper 89-0269, Jan. 1989.

${ }^{16}$ Wright, D., Yotsumata, T., and El-Sheimy, N., "Real Time Identification And Location Of Forest Fire Hotspots From geo-referenced Thermal Images," International Archives of Photogrammetry Remote Sensing and Spatial Information Sciences, Vol. 35, No. 2, 2004, pp. 13-18, also AIAA Paper 89-0269, Jan. 1989. 